\title{
Uma Nota Sobre a Oferta de EILD e Fechamento Vertical do Mercado de Banda Larga no Brasil
}

\author{
Carlos Baigorri, ${ }^{*}$ Wilfredo L. Maldonado ${ }^{\dagger}$
}

\author{
Sumário: 1. Introdução; 2. Estrutura do Mercado de Redes de Transporte de Telecomunicações no Brasil; \\ 3. Evidências e impactos do fechamento vertical; 4. Conclusões; Apêndice. \\ Palavras-chave: Banda Larga, Estrutura de Mercado, Fechamento Vertical. \\ Códigos JEL: $\quad$ L13, L42, L86.
}

\begin{abstract}
O presente trabalho tem o objetivo de analisar a estrutura concorrencial do mercado de redes de transporte de telecomunicações, considerando a oferta de Exploração Industrial de Linhas Dedicadas (EILD). Para tal, são utilizadas informações municipais referentes à oferta de EILD e à demanda por serviços de banda larga em cada município. Após a avaliação da estrutura concorrencial é feita uma análise econométrica de dados referentes à demanda pelos serviços de banda larga em cada município de forma a avaliar a existência de evidências de fechamento vertical nesse mercado.
\end{abstract}

This work aims to analyze the competitive structure of the market for telecommunications transport networks, considering the supply of Leased Lines (EILD). To this end, local information concerning the provision of EILD and the demand for broadband services are used. After identifying the most suitable market structure at that level, we proceed to an econometric analysis of the broadband services demand in each municipality in order to assess the possibility of vertical foreclosure in those markets.

\section{INTRODUÇÃO}

O Brasil tem observado nos últimos anos um crescimento acentuado da participação do acesso à internet em banda larga na vida dos seus cidadãos. Na Figura 1 observamos que entre 2000 e 2012 o crescimento médio do número de acessos em banda larga fixa no Brasil foi de $53 \%$ ao ano nos últimos 12 anos.

Apesar do vigoroso crescimento do acesso à internet em banda larga, dados da Pesquisa Nacional por Amostra de Domicílios (PNAD), realizada pelo IBGE, indicam que em 2012 apenas 40,3\% dos domicílios brasileiros possuíam acesso à internet. De maneira global, esse instrumento não apenas proporciona

\footnotetext{
*Agência Nacional de Telecomunicações - Anatel. E-mail: baigorri@anatel.gov.br

†Universidade Católica de Brasília, Escola de Graduação em Economia. Brasília-DF. E-mail: wilfredo@pos.ucb.br. O autor agradece o apoio financeiro do CNPq através dos processos 304844/2009-8 e 401461/2009-2.
} 
Figura 1. Evolução da base de clientes de banda larga no Brasil.

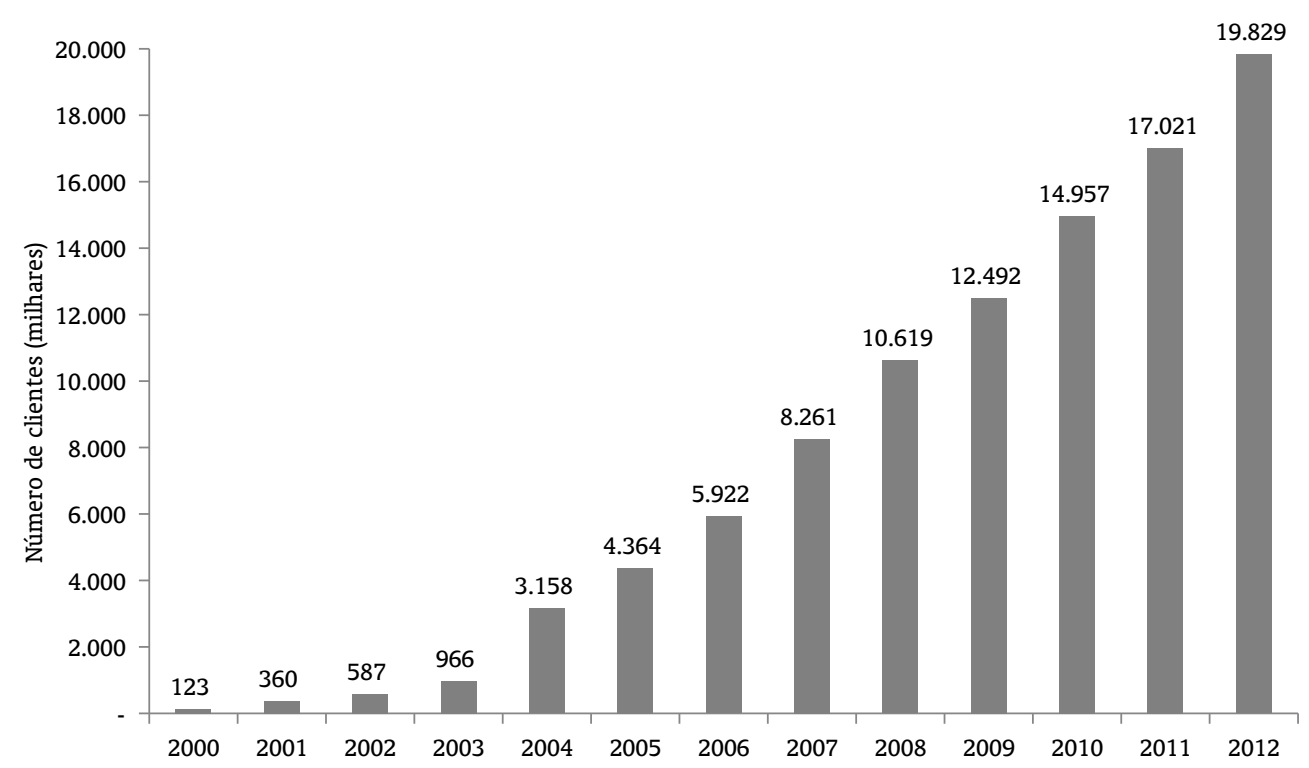

Fonte: Anatel

lazer, mas desenvolvimento econômico. Diversos estudos mostram o impacto do uso da banda larga sobre crescimento do PIB (R. Crandall, Lehr \& Litan, 2007; Czernich, Falck, Kretschmer \& Woessmann, 2009; Koutroumpis, 2009; Qiang, Rossotto \& Kimura, 2009; Thompson \& Garbacz, 2008); outros focam nos impactos do uso da internet sobre a produtividade dos fatores da economia (Waverman, 2009). Finalmente, outros estudos mostram o impacto da adoção da internet na geração de emprego (Atkinson, Castro \& Ezell, 2009; R. W. Crandall, Jackson \& Singer, 2003; Liebenau, Atkinson, Kärrberg, Castro \& Ezell, 2009).

Tendo em vista os benefícios econômicos desse produto, muitos países desenvolveram planos nacionais de banda larga (OCDE, 2011). Seguindo a tendência mundial, em 12 de maio de 2010 o Governo Federal brasileiro publicou o Decreto $\mathrm{n}^{\circ} 7.175$, que institui o Programa Nacional de Banda Larga (PNBL). ${ }^{1}$ Dentre as diversas ações contidas no PNBL, destacamos a proposta de atuação do Governo por meio de uma empresa estatal (Telebras) na oferta de infraestrutura de transporte (transmissão) para a promoção da democratização do acesso à banda larga no Brasil.

A oferta da capacidade de transmissão de dados no atacado, conhecida no mercado como Exploração Industrial de Linhas Dedicadas (EILD), é caracterizada como o serviço no qual uma empresa fornece insumos (linhas dedicadas) para constituição da rede de serviços desta última. Nesse sentido, o acesso à rede de transporte de uma empresa é um dos principais insumos necessários para levar o serviço até o usuário final (redes de acesso), conforme ilustramos na Figura 2.

Um dos argumentos para justificar a atuação governamental na oferta de capacidade de transmissão é a alta concentração desse mercado e a possível integração vertical entre as ofertantes de EILD e as ofertantes de banda larga. Esse tipo de prática anti-competitiva é conhecida na literatura como fechamento vertical (foreclosure). Em Rey \& Tirole (2007) é feita uma ampla revisão da literatura sobre as formas e os efeitos do fechamento de mercados de produtos finais. Na mesma linha, Foros (2004) desenvolve um modelo de mercado de banda larga que prevê duas firmas no mercado de produto final adquirindo o insumo essencial de uma firma monopolista no mercado à montante, integrada verticalmente com uma das firmas.

\footnotetext{
${ }^{1}$ Disponível em www.planalto.gov.br/ccivil_03/_Ato2007-2010/2010/Decreto/D7175.htm
} 
Figura 2. Diagrama de funcionamento das redes de banda larga.

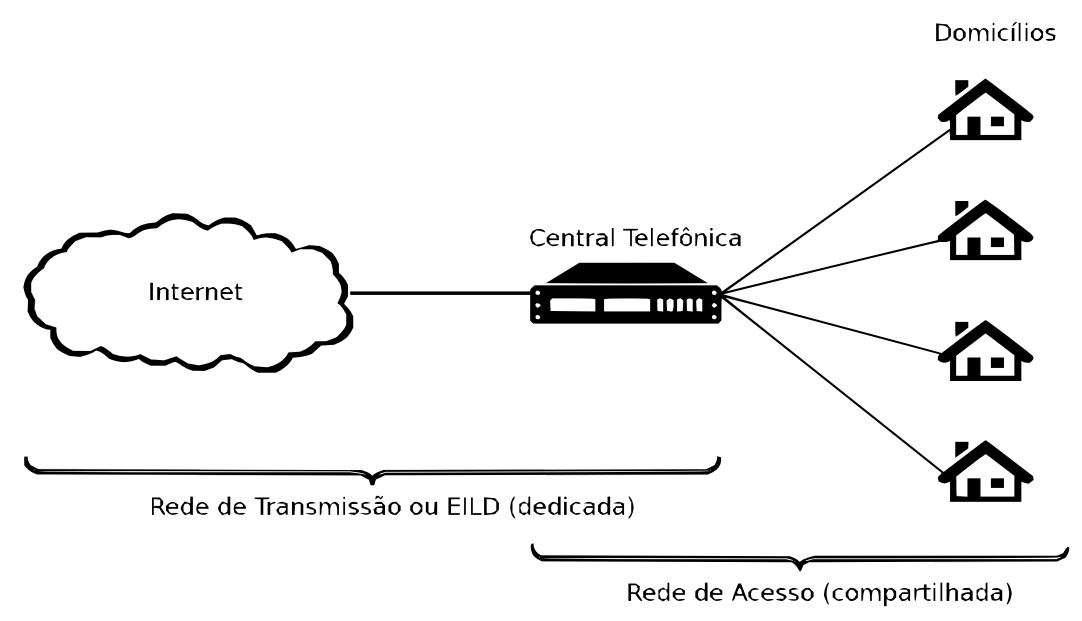

Com base nessa perspectiva de exercício de poder de mercado, o Governo brasileiro propôs a criação de uma empresa estatal (Telebras) para atuar no mercado de acesso à rede de transporte (EILD), reduzindo assim a capacidade de empresas estabelecidas realizarem o fechamento vertical. Nessa proposta, a estratégia anunciada consiste basicamente em ofertar EILD a um preço abaixo do praticado no mercado em uma grande quantidade de municípios.

Com o intuito de proporcionar subsídios para a implementação dessa estratégia por parte do Governo, no presente artigo avaliamos qual das duas estruturas de mercado, Cournot ou Bertrand, é a mais adequada para descrever o mercado de EILD. Finalmente avaliamos a existência de evidências de fechamento vertical do mercado banda larga por parte da posição dominante no mercado de EILD.

O presente trabalho está estruturado da seguinte forma: na seção 2 apresentamos as condições da oferta de EILD no Brasil e identificamos empiricamente qual a estrutura de concorrência imperfeita mais aderente aos dados disponíveis. Na seção 3 mostramos uma análise para avaliar evidências de fechamento vertical. Na seção 4 colocamos nossas considerações finais.

\section{ESTRUTURA DO MERCADO DE REDES DE TRANSPORTE DE TELECOMUNICAÇÕES NO BRASIL}

A exploração das redes de transporte de telecomunicações no Brasil é feita principalmente por meio da oferta de Exploração Industrial de Linhas Dedicadas (EILD). Nesta Seção descreveremos o produto EILD, as suas funcionalidades, os ofertantes de EILD, e utilizaremos um teste original de estrutura de mercado para identificar qual a estrutura concorrencial dessa oferta.

\subsection{0 produto EILD}

A Exploração Industrial de Linhas Dedicadas (EILD) consiste basicamente em um insumo para prestação de serviços de telecomunicações. Esse insumo nada mais é que uma conexão de dados com características técnicas de garantia de taxas de transmissão diferenciadas em relação ao consumidor final. Quando a conexão é entre redes existentes, é necessário que ela tenha características técnicas de garantia contínua de conectividade. Esse tipo de conexão é chamado de linha dedicada, uma vez que não é compartilhada e está integralmente disponível a ambas as redes.

Assim, a EILD é um dos principais insumos para oferta do serviço de acesso à internet em banda larga. Dessa forma, os preços praticados na oferta de acesso à rede de transporte estarão intimamente 
relacionados com os preços praticados na oferta de acesso banda larga (varejo).

No mercado brasileiro tem existido demanda de elementos de rede de transporte para suporte às redes próprias e também a outros serviços prestados por estes operadores no varejo - Serviço Telefônico Fixo Comutado (STFC), Serviço Móvel Pessoal (SMP) e Serviço de Comunicação Multimídia (SCM). Nesse sentido, as empresas de telecomunicações optam por comprar capacidade de transporte, ao invés de terem que construir redes próprias. Atualmente, a oferta de EILD é feita na maior parte pelas concessionárias da telefonia fixa. Essas empresas são monopolistas na oferta de EILD na maioria das cidades brasileiras (87\%), segundo informações da Anatel relativas a aproximadamente 100 mil contratos de EILD.

Duas são as principais componentes do custo fixo das redes de transmissão: o comprimento a capilaridade da rede. Devido à ampla cobertura regional, no Brasil, as redes de transmissão de longa distância são exploradas em monopólio ou duopólio. Analogamente, a necessidade cobertura da rede para diversos pontos aumenta o valor dos investimentos na construção de pontos de agregação de redes. Essa situação é especialmente delicada no Brasil, uma vez que as redes de transmissão chegam a todos os 5.565 municípios brasileiros. A construção de tais redes foi feita na época em que a exploração do serviço era feita pelo Estado. Estes altos custos fixos acompanhados de baixos custos marginais na oferta desse produto fazem com que as redes de transmissão possuam características de monopólio natural no Brasil.

A existência de grupos atuando tanto na rede de transmissão quanto na oferta de banda larga gera condições para um potencial fechamento vertical nesses mercados. Evidência disto é a presença de monopólio na oferta varejista de banda larga em mais de 4.500 municípios brasileiros (aproximadamente de $33 \%$ da população brasileira).

A Figura 3 mostra as consequências desta situação concorrencial. Nele apresentamos a relação entre penetração de banda larga e concentração de mercado em cada um dos 5.565 municípios brasileiros. Como podemos observar, há uma grande quantidade de municípios na região do gráfico associada a uma grande concentração de mercado e uma baixa penetração do serviço.

Figura 3. Dispersão de municípios entre concentração de mercado e penetração de banda larga.

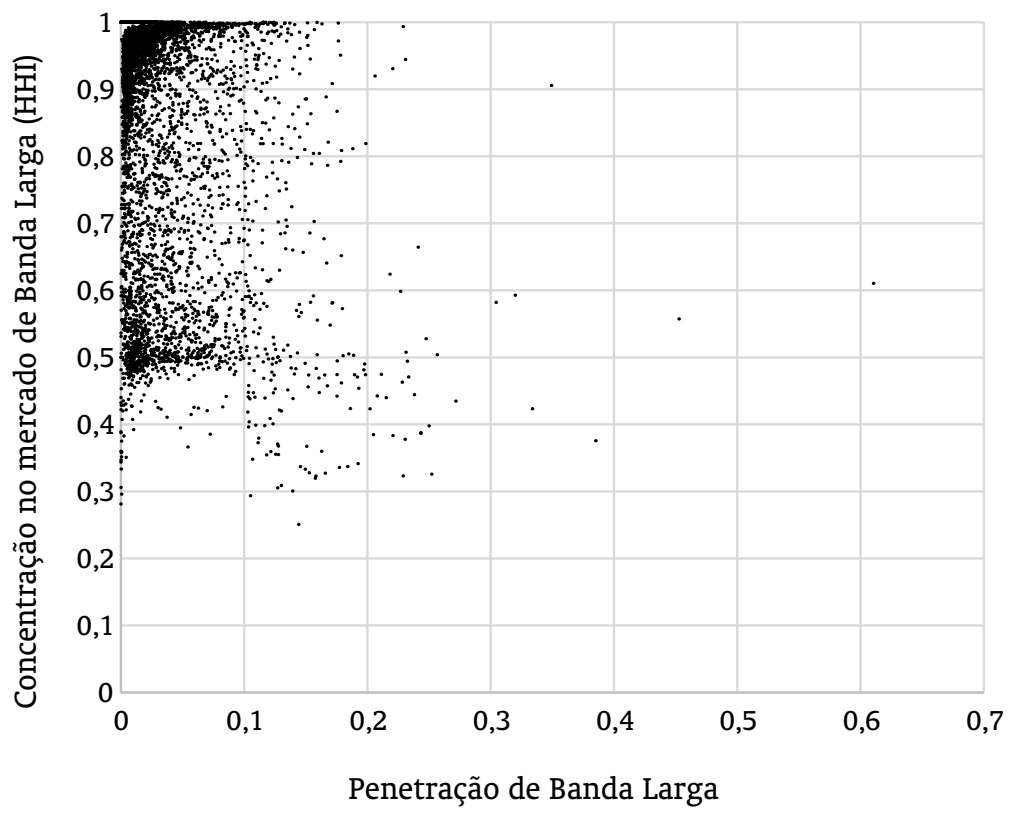

Fonte: Anatel 


\subsection{Ofertantes de EILD}

Por questões históricas vindas do processo de privatização, a demanda tem sido suprida, principalmente, pelas concessionárias do STFC. Contudo, existem outros grupos que ofertam de forma integrada com operações de varejo (GVT, Net Serviços, etc.), e também empresas especializadas na venda desse insumo em relações de atacado (Level 3, Copel Telecomunicações, etc.).

Os preçõs de EILD variam em função da velocidade contratada e da distância entre as redes que se busca conectar. A Figura 4 mostra a distribuição de contratos por taxas de transmissão e a Figura 5 a distribuição de contratos por distância geográfica entre as redes que irá conectar (vão de D0, distância até $5 \mathrm{~km}$, até $\mathrm{D} 8$, distância superior a $1.000 \mathrm{~km}$ ).

Assim, os contratos mais comercializados de EILD são os de 2 Mbps (41.975 contratos) e os de distância D0 (58.477 contratos).

Figura 4. Distribuição dos contratos de EILD em taxas de transmissão.

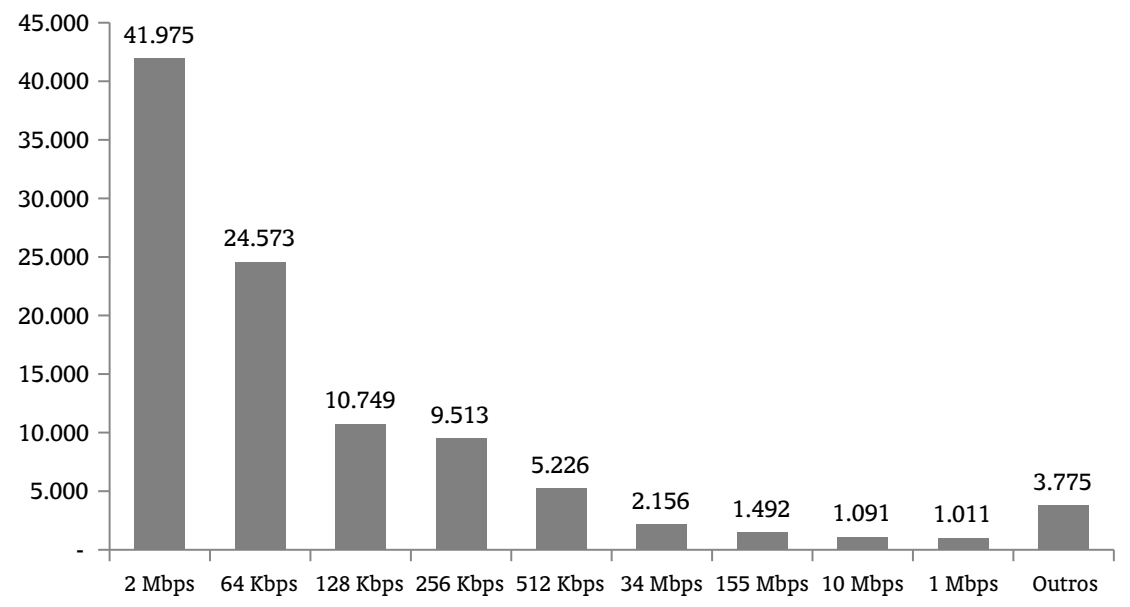

Fonte: Anatel

Figura 5. Distribuição dos contratos de EILD em degraus.

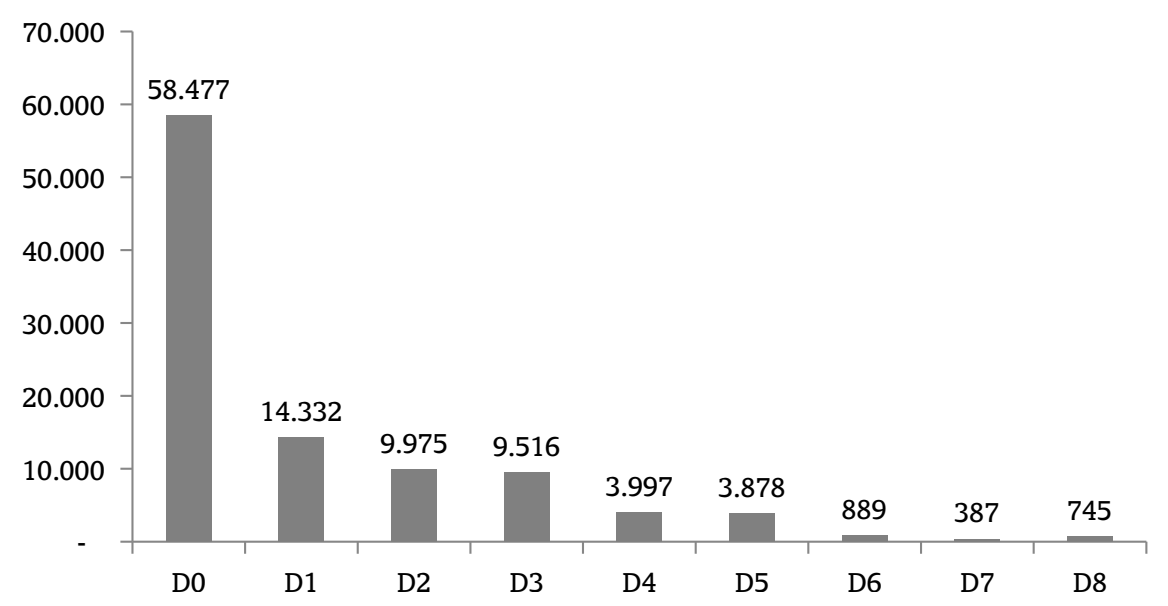

Fonte: Anatel 


\subsection{Estrutura de mercado}

Nesta subseção iremos analisar qual a estrutura de mercado que mais se adequa à competição no nível de EILD no Brasil. Tal análise se justifica em primeiro lugar pois há barreiras econômicas naturais de entrada, dadas pelos altos custos fixos incluídos na construção de redes de transporte.

Logo após a abertura do mercado, a sucessora da estatal privatizada que integrava o Sistema Telebras, dispôs de uma carteira de clientes, de uma marca, e de uma posição já firmada no mercado. Assim, essa vantagem pôde ser aproveitada pelas concessionárias de telefonia fixa (Grupo Oi, Grupo Telefônica e Grupo Embratel). Essas empresas detêm hoje praticamente todas as redes de transporte para oferta de EILD no Brasil. Tanto é assim que essas empresas foram definidas como detentoras de poder de mercado significativo pela Agência Nacional de Telecomunicações.

A Figura 6 mostra que em $78 \%$ dos municípios a oferta de EILD é feita em uma estrutura de monopólio. Ainda, em $16 \%$ dos municípios a estrutura de mercado é de duopólio. Já a Figura 7 mostra que 35,6\% da população brasileira está submetida ao regime de monopólio, e 15,9\% submetida ao regime de duopólio. Assim, concluímos que a competição é mais acirrada nos municípios mais populosos.

Figura 6. Distribuição dos municípios em função da quantidade de ofertantes de EILD.

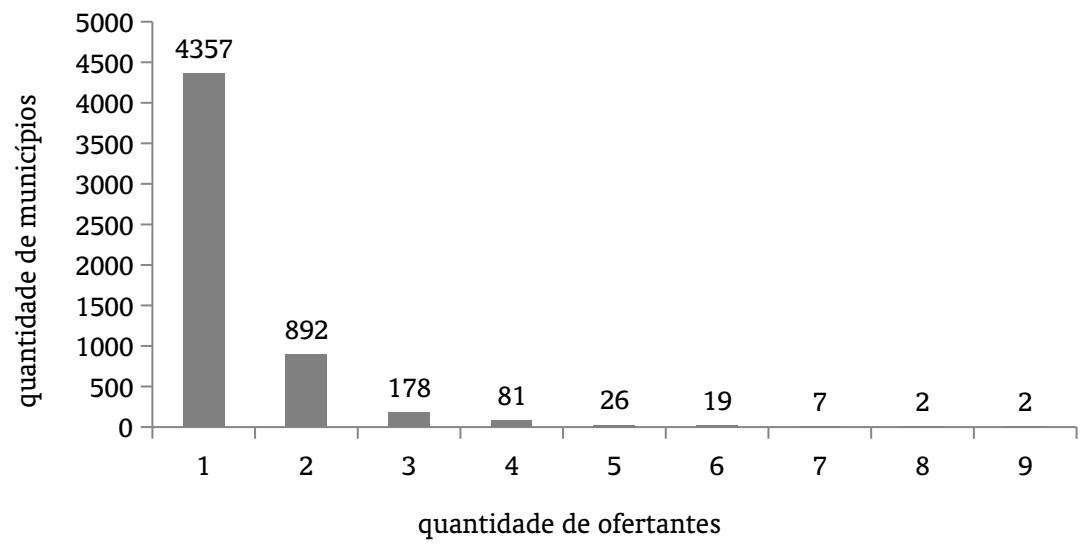

Fonte: Anatel

Figura 7. Distribuição da população em função da quantidade de ofertantes de EILD.

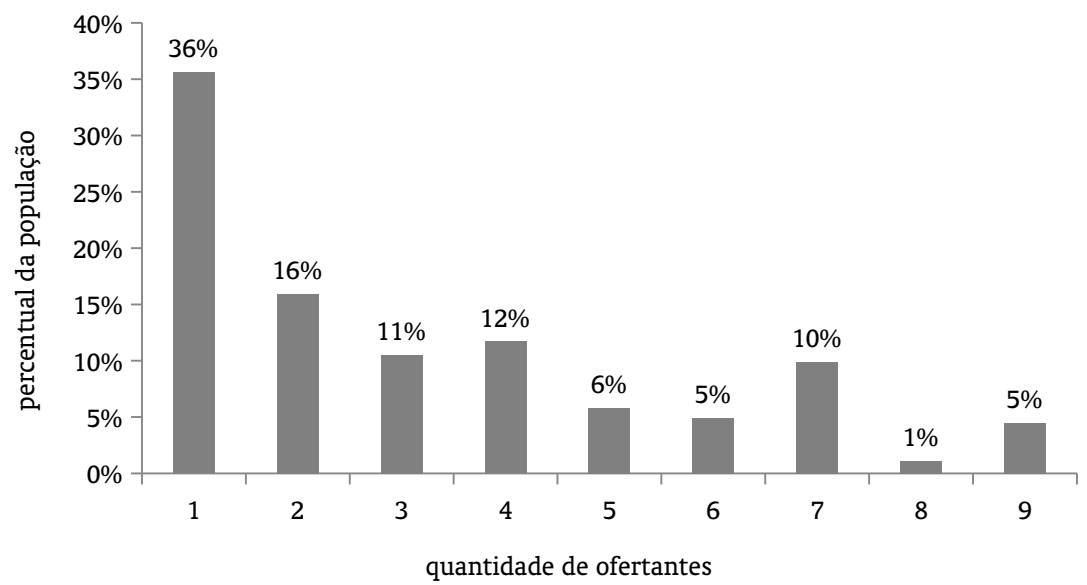

Fonte: Anatel 
Por outro lado, a quantidade de ofertantes em um mercado não necessariamente é um indicador de eficiência nele. Se a concorrência imperfeita for à la Cournot, a ineficiência diminui à medida que novos ofertantes entram no mercado. Entretanto, em uma concorrência imperfeita à la Bertrand com custos simétricos, o nível máximo de eficiência é alcançado com apenas dois ofertantes. Assim, iremos avaliar quais dessas duas estruturas é mais aderente ao mercado de EILD no Brasil.

Outras estruturas de mercado podem ser analisadas, entretanto, a modelagem de fechamento vertical de Rey \& Tirole (2007) prevê apenas essas estruturas nos mercados de insumos. Nessa linha, modelos específicos para o setor de telecomunicações consideram que a oferta de insumos é feita em uma estrutura de Cournot (Faulhaber \& Hogendorn, 2000; Foros, 2004).

Para avaliar a estrutura concorrencial mais aderente ao mercado brasileiro de EILD iremos utilizar como variável explicada $p_{j}$ o preço médio dos contratos da EILD de $2 \mathrm{Mbps}$ local (D0) nos municípios em que existem informações relativas a essas ofertas. Como vimos anteriormente, estes são os contratos mais representativos.

Como variáveis explicativas para esse preço da EILD iremos considerar as seguintes. O número de competidores no mercado, que será a quantidade de ofertantes no município $\left(N_{j}\right)$ ou uma variável binária $\left(B_{j}\right)$ assumindo o valor 1 se o município é atendido por monopólio e 0 em outro caso. A renda das famílias $\left(X_{2, j} \equiv m_{j}\right)$, cuja proxy será o PIB per capita, segundo informações do IBGE. O parâmetro de elasticidade de substituição $\left(X_{3, j} \equiv \delta_{j}\right)$, a ser calculado no Apêndice. O "tamanho" do mercado $\left(X_{4, j} \equiv A_{j}\right)$ dado pelo número de domicílios urbanos permanentes no município, também reportado pelo IBGE. O custo marginal de oferta de EILD, que terá duas componentes: a primeira terá como proxy a área urbana do município $\left(X_{5, j} \equiv R_{j}\right)$ e a segunda será a alíquota do ICMS cobrado no município $\left(X_{6, j} \equiv I_{j}\right)$.

Portanto, iremos estimar dois modelos, alterando tão somente a abordagem referente à quantidade de ofertantes, para averiguar qual explica melhor os dados disponíveis. Assim, caso $N_{j}$ tenha melhor capacidade explicativa em relação a $B_{j}$, teremos então que o modelo de Cournot se adequa melhor, e viceversa. A fundamentação teórica se baseia em que no modelo de Cournot o preço de equilíbrio depende da quantidade de ofertantes no mercado. Já no modelo de Bertrand, o preço de equilíbrio converge para o preço de equilíbrio em concorrência perfeita quando há dois ou mais ofertantes.

Posto isso, iremos estimar os dois modelos seguintes:

$$
\begin{aligned}
& \ln p_{j}=\alpha_{0}+\alpha_{1} N_{j}+\sum_{i=2}^{5} \alpha_{i} \ln X_{i, j}+\epsilon_{j} \\
& \ln p_{j}=\beta_{0}+\beta_{1} B_{j}+\sum_{i=2}^{5} \beta_{i} \ln X_{i, j}+v_{j} .
\end{aligned}
$$

Utilizando dados da Anatel e do IBGE referentes aos 5.565 municípios em 2010 e utilizando o método de mínimos quadrados ordinários (MQO) com correção para heteroscedasticidade, obtemos os resultados na Tabela 1.

A pouca variabilidade da alíquota do ICMS (pois é constante para todos os municípios de uma mesma UF) fez com que essa variável resulte pouco significativa nas duas regressões. Assim, testamos também um modelo sem a inclusão dessa variável.

No Modelo 1, os parâmetros apresentaram sinal de acordo com o modelo de Cournot. Em particular, os preços tendem a ser menores em cidades com maior demanda (coeficientes dos logaritmos de $m, \delta$ e $A$ ). A entrada de um novo competidor no mercado tem uma redução esperada de $3,7 \%$ no preço da EILD. Quanto à proxy de custo, os resultados indicam que a dimensão geográfica dos municípios impacta positivamente no preço da EILD. Observa-se que o modelo especificado se adapta razoavelmente bem aos dados disponíveis $\left(R^{2}=0,617\right)$. Neste e no modelo 2 a heteroscedasticidade foi corrigida pela utilização de erros-padrão robustos e comprovou-se normalidade dos resíduos em todos os quatro modelos estimados utilizando o teste $\chi^{2}$ de Pearson. 
Tabela 1. Resultados das estimativas considerando o modelo de Cournot e de Bertrand.

\begin{tabular}{|c|c|c|c|c|}
\hline \multirow{2}{*}{$\begin{array}{c}\text { Variáveis } \\
\text { Constante }\end{array}$} & \multicolumn{2}{|c|}{ Modelo 1 - Cournot } & \multicolumn{2}{|c|}{ Modelo 2 - Bertrand } \\
\hline & $\begin{array}{r}6,81683^{* * *} \\
(6,45 \mathrm{E}-91)\end{array}$ & $\begin{array}{c}6,76260^{* * *} \\
(4,31 \mathrm{E}-106)\end{array}$ & $\begin{array}{c}7,01466^{* * *} \\
(7,14 \mathrm{E}-101)\end{array}$ & $\begin{array}{c}7,00742^{* * *} \\
(2,63 \mathrm{E}-127)\end{array}$ \\
\hline$m$ & $\begin{array}{c}-0,10357^{* * *} \\
(0,0022)\end{array}$ & $\begin{array}{c}-0,105331^{* * *} \\
(0,0013)\end{array}$ & $\begin{array}{c}-0,12148^{* * *} \\
(0,0002)\end{array}$ & $\begin{array}{c}-0,121693^{* * *} \\
(0,0001)\end{array}$ \\
\hline$\delta$ & $\begin{array}{c}-0,945658^{* * *} \\
(4,26 \mathrm{E}-52)\end{array}$ & $\begin{array}{c}-0,943970^{* * *} \\
(1,02 \mathrm{E}-52)\end{array}$ & $\begin{array}{c}-0,944521^{* * *} \\
(3,74 \mathrm{E}-50)\end{array}$ & $\begin{array}{c}-0,944320^{* * *} \\
(1,73 \mathrm{E}-50)\end{array}$ \\
\hline$A$ & $\begin{array}{c}-0,162861^{* * *} \\
(2,06 \mathrm{E}-12)\end{array}$ & $\begin{array}{c}-0,163174^{* * *} \\
(1,65 \mathrm{E}-12)\end{array}$ & $\begin{array}{c}-0,194065^{* * *} \\
(2,68 \mathrm{E}-19)\end{array}$ & $\begin{array}{c}-0,194090^{* * *} \\
(2,18 \mathrm{E}-19)\end{array}$ \\
\hline$N$ & $\begin{array}{l}-0,0384736^{* * *} \\
(0,009)\end{array}$ & $\begin{array}{c}-0,0379252^{* *} \\
(0,0094)\end{array}$ & $\begin{array}{l}- \\
-\end{array}$ & $\begin{array}{l}- \\
-\end{array}$ \\
\hline$B$ & $\begin{array}{l}- \\
-\end{array}$ & $\begin{array}{l}- \\
-\end{array}$ & $\begin{array}{l}0,0296608 \\
(0,4318)\end{array}$ & $\begin{array}{l}0,0299084 \\
(0,4206)\end{array}$ \\
\hline$I$ & $\begin{array}{l}0,0619748 \\
(0,7191)\end{array}$ & $\begin{array}{l}- \\
-\end{array}$ & $\begin{array}{l}0,00800243 \\
(0,9624)\end{array}$ & $\begin{array}{l}- \\
-\end{array}$ \\
\hline$R$ & $\begin{array}{l}0,0397998^{*} \\
(0,0756)\end{array}$ & $\begin{array}{l}0,0406847^{*} \\
(0,0643)\end{array}$ & $\begin{array}{l}0,0418908^{*} \\
(0,0555)\end{array}$ & $\begin{array}{l}0,0420043^{*} \\
(0,05)\end{array}$ \\
\hline$R^{2}$ (adj.) & 0,6161 & 0,6168 & 0,6097 & 0,6106 \\
\hline$F$ & $\begin{array}{l}72,478 \\
(4,98 \mathrm{E}-61)\end{array}$ & $\begin{array}{l}87,116 \\
(5,53 \mathrm{E}-62)\end{array}$ & $\begin{array}{l}70,767 \\
(5,84 \mathrm{E}-60)\end{array}$ & $\begin{array}{l}85,079 \\
(6,39 \mathrm{E}-61)\end{array}$ \\
\hline Observações & 406 & 406 & 406 & 406 \\
\hline
\end{tabular}

Notas: Valores entre parênteses representam o $p$-valor das estimativas. ${ }^{* * *},{ }^{* *} \mathrm{e}{ }^{*}$ indicam estimativas significantes ao nível de $1 \%, 5 \%$ e $10 \%$, respectivamente.

Em relação ao Modelo 2, todos os parâmetros estimados apresentaram sinal coerente com o modelo de Bertrand. Também aqui os resultados indicam que os preços tendem a ser menores em cidades com maior demanda (coeficientes dos logaritmos de $m, \delta$ e $A$ ). Por outro lado, a estimativa dos efeitos da competição (coeficiente de $B$ ) apresenta sinal de acordo do esperado, porém sem significância estatística. Novamente, o modelo especificado se adapta razoavelmente bem aos dados disponíveis $\left(R^{2}=0,611\right)$.

Comparando os resultados dos dois modelos concluímos que a competição no mercado de EILD apresenta uma estrutura competitiva mais aderente ao modelo de Cournot. Essa conclusão faz sentido se temos em vista que a EILD é oferecida por meio de redes de telecomunicações e que a capilaridade dessa rede (e portanto, a dimensão geográfica) acaba sendo um fator determinante. Assim, a decisão de cada firma está associada à quantidade de endereços que podem ser atendidos, gerando-se uma competição mais aderente à de Cournot.

A Figura 8 corrobora empiricamente a conclusão acima. Nele o preço máximo para EILD é função da quantidade de ofertantes em cada município. Há uma evidente redução do preço da EILD quando aumenta a quantidade de competidores, observando-se uma certa estabilidade a partir da entrada do sexto ofertante. 
Figura 8. Relação entre quantidade de ofertantes e preços de EILD.

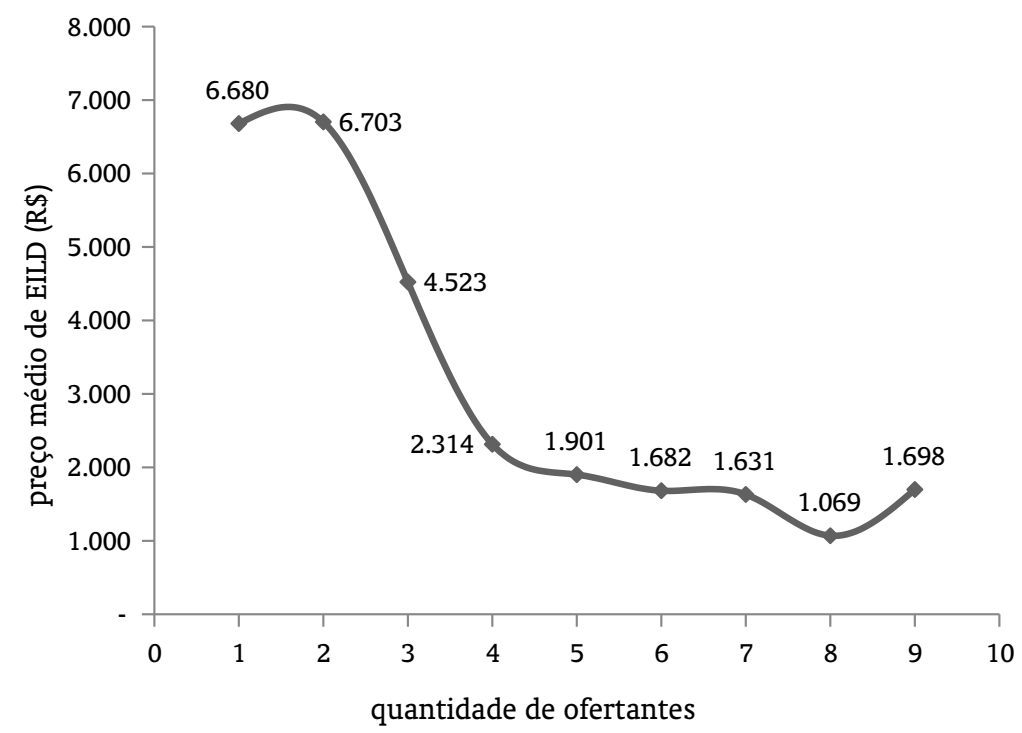

Fonte: Anatel

\section{EVIDÊNCIAS E IMPACTOS DO FECHAMENTO VERTICAL}

Nesta seção iremos avaliar evidências de fechamento vertical nos mercados de EILD e de banda larga através de um aprimoramento da análise feita por Macedo \& Carvalho (2010). Na Figura 9 podemos observar que tanto a concentração no mercado de banda larga quanto o preço praticado pelos contratos de EILD estão negativamente correlacionados com o número de competidores no mercado de EILD. Isto é uma forte evidência de fechamento vertical entre esses mercados.

Conforme podemos observar, os níveis de concentração do mercado de banda larga (downstream) tendem a ser maiores nos municípios em que não há competição na oferta de EILD (upstream), ou seja, nas cidades em que a rede de transporte é um gargalo para os ofertantes de banda larga. Isto está de acordo com o previsto na literatura sobre fechamento vertical (Rey \& Tirole, 2007).

A prática do fechamento vertical pode ter um efeito sobre a penetração do serviço de banda larga nos municípios. O poder de mercado na oferta de EILD é trasferido ao mercado do produto final, gerando assim uma quantidade demandada pela banda larga que está aquém daquela num cenário sem o fechamento vertical.

Os determinantes da penetração da banda larga nos municípios brasileiros foram investigados em Macedo \& Carvalho (2010). Usando dados municipais, eles identificam quais os possíveis determinantes da penetração da banda larga. Nesse contexto, utilizaram como variáveis explicativas o nível de concentração do mercado de banda larga, bem como o nível de competição entre diferentes plataformas tecnológicas, conforme Distaso, Lupi \& Manenti (2006). ${ }^{2}$

A conclusão de Macedo e Carvalho (Macedo \& Carvalho, 2010, p.26) é que "quanto maior for a disponibilidade de tecnologias de prestação do serviço de banda larga no município, maior será a competição, o que terá influência positiva sobre o aumento da penetração do serviço." Entretanto, destacam os autores que:

\footnotetext{
${ }^{2}$ Eles consideram dois tipos de tecnologia de transmissão: as redes DSL que proporcionam banda larga por meio da rede de telefonia convencional e as redes HFC que o fazem por meio de redes de TV a cabo.
} 
Figura 9. Relação entre quantidade de ofertantes, preços de EILD e concentração de mercado.

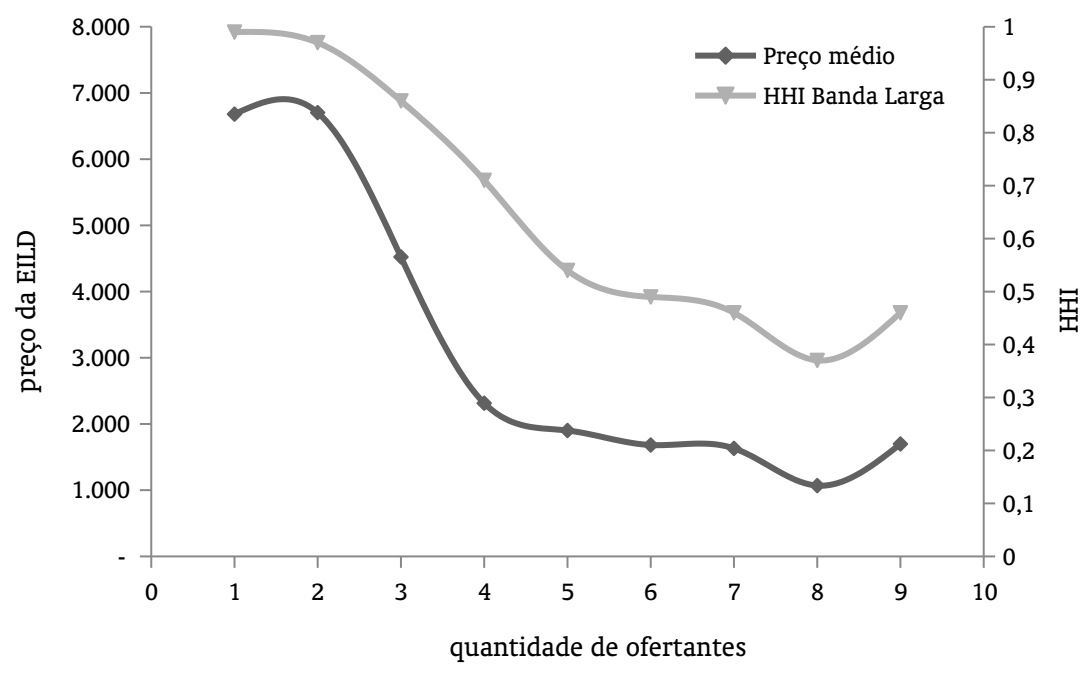

Fonte: Anatel

[...] houve resultados que não corresponderam ao esperado. As análises de regressão, neste caso específico, utilizando-se este conjunto de dados disponíveis em particular, não indicaram um relacionamento positivo entre aumento da competição entre empresas e aumento da penetração do serviço. Talvez isto se explique pelo alto grau de concentração econômica na exploração do serviço ao nível do município. (Macedo \& Carvalho, 2010, p.26)

Assim, iremos avaliar evidências de fechamento vertical entre o mercado de EILD e o de banda larga incorporando ao modelo Macedo \& Carvalho (2010) a quantidade de ofertantes de EILD e o preço médio da EILD nos municípios, como variáveis explicativas. Faremos isto utilizando dados mais atuais dos municípios (dados de 2010).

Especificamente, sejam $X_{i}$ o conjunto de variáveis de controle; estimaremos os seguintes modelos:

$$
\begin{aligned}
& \ln \text { Penet }_{j}=\alpha_{0}+\alpha_{i} \ln X_{i}+\beta_{1} \ln \mathrm{HHI}_{\mathrm{emp}, j}+\epsilon_{j} \\
& \ln \text { Penet }_{j}=\alpha_{0}+\alpha_{i} \ln X_{i}+\beta_{1} \ln \mathrm{HHI}_{\mathrm{tec}, j}+\epsilon_{j} \\
& {\ln \text { Penet }_{j}}=\alpha_{0}+\alpha_{i} \ln X_{i}+\beta_{1} \ln \mathrm{HHI}_{\mathrm{emp}}+\beta_{2} \ln _{\mathrm{HHI}}, j+\epsilon_{j} \\
& {\ln \text { Penet }_{j}}=\alpha_{0}+\alpha_{i} \ln X_{i}+\beta_{1} N_{j}+\epsilon_{j} \\
& \ln \text { Penet }_{j}=\alpha_{0}+\alpha_{i} \ln X_{i}+\beta_{1} \ln \mathrm{EILD}_{j}+\epsilon_{j} \\
& {\ln \text { Penet }_{j}}=\alpha_{0}+\alpha_{i} \ln X_{i}+\beta_{1} \ln \mathrm{EILD}_{j}+\beta_{2} \ln \mathrm{HHI}_{\mathrm{tec}, j}+\epsilon_{j}
\end{aligned}
$$

Na Tabela 2 apresentamos a definição de cada variável dos modelos e as fontes, incluindo as variáveis de controle utilizadas em Macedo \& Carvalho (2010).

A Tabela 3 apresenta os resultados das estimações. Por causa da disponibilidade de dados referentes a preços de EILD, os modelos 5 e 6 foram rodados com 406 amostras e os outros com 5.312. Todos os modelos foram estimados utilizando correção para heteroscedasticidade.

Como em Macedo \& Carvalho (2010), as estimativas dos parâmetros associados à competição entre empresas têm sinais contrários aos esperados nos modelos 1 e 3 . Já as estimativas referentes à concentração de mercado entre plataformas tecnológicas têm o sinal esperado nos modelos 2 , 3 e 6 . Entretanto, no modelo 6, quando incluímos informações sobre preços de EILD percebemos que esse parâmetro não tem significância estatística. Dessa forma, o modelo 6 indica que a competição entre plataformas não é 
Tabela 2. Descrição das variáveis utilizadas.

\begin{tabular}{llc}
\hline Variável & Definição & Fonte \\
\hline Penet & Penetração da banda larga no município & Anatel \\
\%Agro & Percentual do PIB do município decorrente de atividades agropecuárias & IBGE \\
\%Ind. & Percentual do PIB do município decorrente de atividades industriais & IBGE \\
\%Serv. & Percentual do PIB do município decorrente de atividades de prestação & IBGE \\
& de serviços & FIRJAN \\
IFDM_Edu & Índice FIRJAN de desenvolvimento da educação & FIRJAN \\
IFDM_Emp & Índice FIRJAN de desenvolvimento do emprego e renda & FIRJAN \\
IFDM_Saude & Índice FIRJAN de desenvolvimento da saúde & IBGE \\
DPPU & Quantidade de Domicílios Permanentes Urbanos & IBGE \\
PIBpc & PIB per capita & Anatel \\
HHI_empresas & HHI do mercado de banda larga & Anatel \\
HHI_tecnologias & HHI entre as tecnologias DSL e HFC & Anatel \\
$N$ & Quantidade de ofertantes de EILD & Anatel \\
EILD & Preço médio da EILD &
\end{tabular}

Tabela 3. Resultados das estimativas dos modelos 1 a 6 .

\begin{tabular}{|c|c|c|c|c|c|c|}
\hline Variáveis & Modelo 1 & Modelo 2 & Modelo 3 & Modelo 4 & Modelo 5 & Modelo 6 \\
\hline Constante & $\begin{array}{r}-10,2549^{* * *} \\
(1,53 \mathrm{E}-120)\end{array}$ & $\begin{array}{l}-10,1962^{* * *} \\
(3,50 \mathrm{E}-121)\end{array}$ & $\begin{array}{l}-10,2368^{* * *} \\
\quad(1,40 \mathrm{E}-121)\end{array}$ & $\begin{array}{c}-9,88881^{* * *} \\
(1,97 \mathrm{E}-116)\end{array}$ & $\begin{array}{c}-3,46345^{* * *} \\
(6,00 \mathrm{E}-4)\end{array}$ & $\begin{array}{c}-3,37006^{* * *} \\
(9,00 \mathrm{E}-4)\end{array}$ \\
\hline \%Agro & $\begin{array}{l}0,00245631 \\
(0,8658)\end{array}$ & $\begin{array}{l}0,0087553 \\
(0,5445)\end{array}$ & $\begin{array}{l}0,00579605 \\
(0,6899)\end{array}$ & $\begin{array}{l}0,0212209 \\
(0,1427)\end{array}$ & $\begin{array}{c}-0,0031397 \\
(0,8381)\end{array}$ & $\begin{array}{c}-0,00407076 \\
(0,7976)\end{array}$ \\
\hline \%Ind. & $\begin{array}{l}0,154058^{* * *} \\
(1,09 \mathrm{E}-8)\end{array}$ & $\begin{array}{l}0,162405^{* * *} \\
(1,52 \mathrm{E}-9)\end{array}$ & $\begin{array}{l}0,158383^{* * *} \\
(4,29 \mathrm{E}-9)\end{array}$ & $\begin{array}{c}0,190167^{* * *} \\
(2,37 \mathrm{E}-12)\end{array}$ & $\begin{array}{c}-0,0472566 \\
(4,82 \mathrm{E}-1)\end{array}$ & $\begin{array}{c}-0,0424913 \\
(5,32 \mathrm{E}-1)\end{array}$ \\
\hline \%Serv. & $\begin{array}{c}0,788711^{* * *} \\
(1,45 \mathrm{E}-20)\end{array}$ & $\begin{array}{c}0,798151^{* * *} \\
(3,19 \mathrm{E}-21)\end{array}$ & $\begin{array}{c}0,795391^{* * *} \\
(4,95 \mathrm{E}-21)\end{array}$ & $\begin{array}{c}0,829030^{* * *} \\
(1,07 \mathrm{E}-23)\end{array}$ & $\begin{array}{c}0,130174 \\
(3,30 \mathrm{E}-1)\end{array}$ & $\begin{array}{c}0,146609 \\
(2,80 \mathrm{E}-1)\end{array}$ \\
\hline IFDM_Edu & $\begin{array}{r}1,87253^{* * *} \\
(5,98 \mathrm{E}-51)\end{array}$ & $\begin{array}{r}1,85183^{* * *} \\
(4,19 \mathrm{E}-50)\end{array}$ & $\begin{array}{r}1,84210^{* * *} \\
(2,07 \mathrm{E}-49)\end{array}$ & $\begin{array}{r}1,938666^{* * *} \\
(6,07 \mathrm{E}-55)\end{array}$ & $\begin{array}{r}2,49634^{* * *} \\
(1,19 \mathrm{E}-12)\end{array}$ & $\begin{array}{r}2,44814^{* * *} \\
(1,58 \mathrm{E}-11)\end{array}$ \\
\hline IFDM_Emp & $\begin{array}{l}0,111263^{* *} \\
(0,0206)\end{array}$ & $\begin{array}{l}0,107457^{* *} \\
(0,0248)\end{array}$ & $\begin{array}{l}0,110792^{* *} \\
(0,0208)\end{array}$ & $\begin{array}{l}0,0937472^{* *} \\
(0,0482)\end{array}$ & $\begin{array}{l}0,452417^{* * *} \\
(0,0027)\end{array}$ & $\begin{array}{l}0,448770^{* * *} \\
(0,003)\end{array}$ \\
\hline IFDM_Saude & $\begin{array}{l}0,561779 * * * \\
(0,0001)\end{array}$ & $\begin{array}{l}0,560605^{* * *} \\
(0,0001)\end{array}$ & $\begin{array}{l}0,570675^{* * *} \\
(7,88 \mathrm{E}-5)\end{array}$ & $\begin{array}{l}0,458523^{* * *} \\
(1,40 \mathrm{E}-3)\end{array}$ & $\begin{array}{l}1,67362^{* *} \\
(0,0145)\end{array}$ & $\begin{array}{l}1,65823^{* *} \\
(0,0169)\end{array}$ \\
\hline DPPU & $\begin{array}{c}0,191696^{* * *} \\
(2,74 \mathrm{E}-34)\end{array}$ & $\begin{array}{c}0,187367^{* * *} \\
(2,67 \mathrm{E}-34)\end{array}$ & $\begin{array}{c}0,191251^{* * *} \\
(3,13 \mathrm{E}-34)\end{array}$ & $\begin{array}{c}0,154155^{* * *} \\
(4,08 \mathrm{E}-22)\end{array}$ & $\begin{array}{c}0,122469^{* * *} \\
(0,0000591)\end{array}$ & $\begin{array}{l}0,110775^{* * *} \\
(0,0021)\end{array}$ \\
\hline PIBpc & $\begin{array}{c}0,786242^{* * *} \\
(2,94 \mathrm{E}-61)\end{array}$ & $\begin{array}{c}0,785919^{* * *} \\
(5,61 \mathrm{E}-62)\end{array}$ & $\begin{array}{c}0,786087^{* * *} \\
(5,20 \mathrm{E}-62)\end{array}$ & $\begin{array}{c}0,765480^{* * *} \\
(2,22 \mathrm{E}-61)\end{array}$ & $\begin{array}{l}0,305879^{* * *} \\
(1,10 \mathrm{E}-3)\end{array}$ & $\begin{array}{l}0,303634^{* * *} \\
(1,20 \mathrm{E}-3)\end{array}$ \\
\hline HHI_empresas & $\begin{array}{c}-0,156712 \\
(0,11)\end{array}$ & $\begin{array}{l}- \\
-\end{array}$ & $\begin{array}{l}0,229399 \\
(0,1431)\end{array}$ & $\begin{array}{l}- \\
-\end{array}$ & $\begin{array}{l}- \\
-\end{array}$ & $\begin{array}{l}- \\
-\end{array}$ \\
\hline HHI_tecnologias & $\begin{array}{l}- \\
-\end{array}$ & $\begin{array}{c}-0,604683^{* * *} \\
(2,45 \mathrm{E}-10)\end{array}$ & $\begin{array}{c}-0,825877^{* * *} \\
(3,25 \mathrm{E}-6)\end{array}$ & $\begin{array}{l}- \\
-\end{array}$ & $\begin{array}{l}- \\
-\end{array}$ & $\begin{array}{c}-0,125352 \\
(0,3298)\end{array}$ \\
\hline$N$ & $\begin{array}{l}- \\
-\end{array}$ & $\begin{array}{l}- \\
-\end{array}$ & $\begin{array}{l}- \\
-\end{array}$ & $\begin{array}{c}0,177779 * * * \\
(3,03 \mathrm{E}-25)\end{array}$ & $\begin{array}{l}- \\
-\end{array}$ & $\begin{array}{l}- \\
-\end{array}$ \\
\hline EILD & $\begin{array}{l}- \\
-\end{array}$ & $\begin{array}{l}- \\
-\end{array}$ & - & $\begin{array}{l}- \\
-\end{array}$ & $\begin{array}{l}-0,175981^{* * *} \\
(0,01)\end{array}$ & $\begin{array}{c}-0,172258^{* *} \\
(0,0132)\end{array}$ \\
\hline$R^{2}$ (adj.) & 0,5067 & 0,5079 & 0,508 & 0,5145 & 0,5727 & 0,5724 \\
\hline$F$ & $\begin{array}{l}741,675 \\
(0)\end{array}$ & $\begin{array}{l}905,602 \\
(0)\end{array}$ & $\begin{array}{l}810,49 \\
(0)\end{array}$ & $\begin{array}{l}842,467 \\
(0)\end{array}$ & $\begin{array}{l}39,015 \\
(1,82 \mathrm{E}-49)\end{array}$ & $\begin{array}{l}51,436 \\
(2,08 \mathrm{E}-65)\end{array}$ \\
\hline Observações & 5312 & 5312 & 5312 & 5312 & 406 & 406 \\
\hline
\end{tabular}

Notas: Valores entre parênteses representam o $p$-valor das estimativas. ${ }^{* *},{ }^{* *} \mathrm{e}^{*}$ indicam estimativas significantes ao nível de $1 \%$, $5 \%$ e $10 \%$, respectivamente. 
um fator determinante da penetração da banda larga, mas que o preço da EILD sim. Isto está em linha com a hipótese de fechamento vertical.

Quando avaliamos a utilização dos preços médios de EILD, identificamos que nos modelos 5 e 6 as estimativas têm sinal de acordo com o esperado, além de terem significância estatística. Assim, podemos concluir que a reduzida quantidade de ofertantes de redes de transporte e o consequente elevado preço da EILD afeta de forma negativa a adoção da banda larga pelas famílias brasileiras.

Assim, a Figura 9 junto com os resultados dos modelos econométricos (em especial, do modelo 6) trazem indícios de que há prática de fechamento vertical no mercado de telecomunicações. Além disso, nos permite mensurar os impactos da elevação do preço da EILD sobre a penetração da banda larga na população.

\section{CONCLUSÕES}

Os altos custos fixos associados à construção de redes de transporte de dados tornam o mercado de banda larga um mercado com alta concentração. Dessa forma, é fundamental a identificação da estrutura de concorrência nesse mercado para poder definir políticas efetivas de massificação do acesso à banda larga.

Por outro lado, a EILD é um insumo essencial para prestação de serviços de banda larga, e na maioria das cidades há um monopólio na oferta desse produto. Como empresas que ofertam EILD também podem atuar no mercado final de banda larga, temos as condições propícias para a prática de fechamento vertical.

Neste trabalho propomos uma análise econométrica da estrutura de mercado de banda larga; especificamente, analisamos qual das duas estruturas de mercado, Cournot ou Bertrand, é a mais aderente aos dados levantados dos 5.565 municípios brasileiros. Os resultados indicam que a estrutura de Cournot é a mais adequada para modelar esse mercado. A identificação dessa estrutura concorrencial está alinhada com as características técnicas do provimento do produto da EILD. De fato, tendo em vista que a oferta da EILD é uma oferta que envolve a construção de uma infraestrutura, é de se esperar que a estrutura concorrencial seja mais aderente a uma onde empresas escolhem a quantidade a ofertar (i.e. o tamanho de suas redes) e não o preço do seu produto.

Finalmente realizamos uma análise dos determinantes da penetração do serviço de banda larga nos municípios brasileiros e encontramos que o preço do produto gerado pelo mercado à montante (EILD) é um determinante que afeta negativamente a penetração da banda larga na economia. Isto é uma evidência (embora não uma prova contundente) do fechamento vertical destes dois mercados.

Desta maneira, fica clara a necessidade de políticas públicas que evitem a possibilidade do fechamento vertical, ou que minimizem os seus efeitos sobre o mercado. Uma possibilidade é a atuação do Estado gerando um oligopólio misto na oferta de EILD e na promoção do uso da banda larga. Fica ainda esse desafio, dada a ausência de consenso na literatura internacional sobre a forma mais eficaz de intervir nesse mercado.

\section{REFERÊNCIAS BIBLIOGRÁFICAS}

Atkinson, R. D., Castro, D. \& Ezell, S. J. (2009, January). The digital road to recovery: A stimulus plan to create jobs, boost productivity and revitalize America (Relatório técnico). Washington, DC: The Information Technology and Innovation Foundation (ITIF). Disponível em: http://www.areadevelopment.com/article _pdf/id9806_roadtorecovery.pdf

Crandall, R., Lehr, W. \& Litan, R. (2007, July). The effects of broadband deployment on output and employment: A cross-sectional analysis of U.S. data (Issues in Economic Policy $\mathrm{N}^{0}$ 6). The Brookings Institution. Disponível em: http://www.brookings.edu/research/papers/2007/06/labor-crandall

Crandall, R. W., Jackson, C. L. \& Singer, H. J. (2003, September). The effect of ubiquitous broadband adoption on investment, jobs, and the U.S. economy (Relatório técnico). Washington, DC: Criterion Economics L.L.C. 
Czernich, N., Falck, O., Kretschmer, T. \& Woessmann, L. (2009, December). Broadband infrastructure and economic growth (CESifo Working Paper $\mathrm{N}^{0}$ 2861). CESifo (Center for Economic Studies and Ifo Institute for Economic Research). Disponível em: www.ifo.de/DocCIDL/cesifo1_wp2861.pdf

Distaso, W., Lupi, P. \& Manenti, F. M. (2006). Platform competition and broadband uptake: Theory and empirical evidence from the European Union. Information Economics and Policy, 18(1), 87-106. doi: 10.1016/j.infoecopol.2005.07.002

Faulhaber, G. R., \& Hogendorn, C. (2000). The market structure of broadband telecommunications. The Journal of Industrial Economics, 48(3), 305-329. doi: 10.1111/1467-6451.00125

Foros, Ø. (2004). Strategic investments with spillovers, vertical integration and foreclosure in the broadband access market. International Journal of Industrial Organization, 22(1), 1-24. doi: 10.1016/S0167-7187(03)00079-1

Koutroumpis, P. (2009). The economic impact of broadband on growth: A simultaneous approach. Telecommunications Policy, 33(9), 471-485. doi: 10.1016/j.telpol.2009.07.004

Liebenau, J., Atkinson, R., Kärrberg, P., Castro, D. \& Ezell, S. (2009, April). The UK's digital road to recovery (Relatório técnico). London School of Economics and Political Science (LSE) and the Information Technology and Innovation Foundation (ITIF). Disponível em: http://www.itif.org/files/digitalrecovery.pdf

Macedo, H. R., \& Carvalho, A.X.Y.d. (2010). Análise de possíveis determinantes da penetração do serviço de acesso à internet em banda larga nos municípios brasileiros (Textos Para Discussão $\mathrm{N}^{0}$ 1503). Instituto de Pesquisas Econômicas Aplicadas - IPEA. Disponível em: http://hdl.handle.net/11058/2978

OCDE. (2011). National broadband plans (OECD Digital Economy Papers $\mathrm{N}^{0}$ 181). OCDE (DSTI/ICCP/CISP). doi: $10.1787 / 5 \mathrm{~kg} 9 \mathrm{sr} 5 \mathrm{fmqwd}$-en

Qiang, C. Z.-W., Rossotto, C. M. \& Kimura, K. (2009). Economic impacts of broadband. In T. W. Bank (Ed.), Information and communications for development 2009: Extending reach and increasing impact (pp. 35-50). The World Bank. Disponível em: http://go.worldbank.org/NATLOH7HV0

Rey, P., \& Tirole, J. (2007). A primer on foreclosure. In M. Armstrong \& R. Porter (Eds.), Handbook of industrial organization (Vol. 3, pp. 2145-2220). Elsevier. doi: 10.1016/S1573-448X(06)03033-0

Thompson, H., \& Garbacz, C. (2008). Broadband impacts on state GDP: Direct and indirect impacts. Canada. (Paper apresentado no International Telecommunications Society 17th Biennial Conference)

Waverman, L. (2009). Economic impact of broadband: An empirical study. London: LECG.

\section{APÊNDICE.}

Calibragem da elasticidade de substituição do serviço de banda larga

A utilidade da $n$-ésima família do município $i$ é

$$
u_{n, i}(x, m)=\left[\alpha_{n} x^{\delta_{i}}+\left(1-\alpha_{n}\right) m^{\delta_{i}}\right]^{1 / \delta_{i}},
$$

onde $x \in\{0,1\}$ é a decisão de contratação de serviços de banda larga e $m \geq 0$ representa os outros gastos. A elasticidade de substituição entre serviços de banda larga e renda é $\left(1-\delta_{i}\right)^{-1}$ e $\alpha_{n}$ é o peso do gasto com serviços de banda larga no orçamento da família.

Para calibrar o parâmetro $\delta_{i}$ procedemos da seguinte maneira: Utilizando dados da Pesquisa de Orçamentos Familiares (POF), ordenamos as famílias de maneira decrescente na renda. Utilizando dados da Anatel, calculamos a última família mais rica no município que contrata serviços de banda larga. Seja $m_{n, i}$ a renda dessa família e $p_{i}$ o preço da banda larga no município $i$. Então calbramos o parâmetro $\delta_{i}$ como aquele que satisfaz $u_{n, i}\left(1, m_{n, i}-p_{i}\right)=u_{n, i}\left(0, m_{n, i}\right)$, ou seja, o que resolve a equação

$$
\frac{\alpha_{n}}{1-\alpha_{n}}=m_{n, i}^{\delta_{i}}-\left(m_{n, i}-p_{i}\right)^{\delta_{i}}
$$

Assim, o parâmetro que define a elasticidade é aquele que deixa indiferente entre ter e não ter esse serviço à última família mais rica que contrata o serviço. A seguir, mostramos a existência e unicidade da solução à equação acima. 
Existência: Seja $f(x)=a^{x}-b^{x}$, onde $a>b>1$. Em nosso caso $a=m_{n, i}$ e $b=m_{n, i}-p_{i}$. Como $f(x)=$ $b^{x}\left[\left(a b^{-1}\right)^{x}-1\right]$, então $f(0)=0$ e $f(+\infty)=+\infty$. Portanto sempre existe $x_{0}$ tal que $f\left(x_{0}\right)=\alpha /(1-\alpha)$.

Unicidade: A derivada $f^{\prime}(x)=a^{x} \ln a-b^{x} \ln b$ é estritamente positiva para todo $x>0$. Para verificar isto basta ver que $(a / b)>1$ e $\frac{\ln b}{\ln a}<1$; então $(a / b)^{x}>\frac{\ln b}{\ln a}$ e portanto $f^{\prime}(x)>0$.

Para calibrar $\alpha_{n}$ na cidade $i$ utilizamos informações da POF para calcular o percentual da renda das famílias gasto com serviço de telecomunicações. $O$ parâmetro tem dimensão geográfica (associada à região do município) e dimensão de classe de renda. A Tabela A-1 mostra os valores considerados.

Tabela A-1. Parâmetro $\alpha_{n}$ para cada classe de renda e região geográfica.

\begin{tabular}{lcccccc}
\hline & \multicolumn{6}{c}{ Classe de renda } \\
\cline { 2 - 7 } Região & $\mathrm{F}$ & $\mathrm{E}$ & $\mathrm{D}$ & $\mathrm{C}$ & $\mathrm{B}$ & $\mathrm{A}$ \\
\hline Sul & $0,20 \%$ & $0,20 \%$ & $0,40 \%$ & $0,70 \%$ & $0,80 \%$ & $0,70 \%$ \\
Sudeste & $0,10 \%$ & $0,20 \%$ & $0,50 \%$ & $0,70 \%$ & $1,00 \%$ & $0,80 \%$ \\
Centro-Oeste & $0,30 \%$ & $0,30 \%$ & $0,40 \%$ & $0,70 \%$ & $0,90 \%$ & $0,80 \%$ \\
Nordeste & $0,10 \%$ & $0,10 \%$ & $0,10 \%$ & $0,40 \%$ & $0,70 \%$ & $0,50 \%$ \\
Norte & $0,10 \%$ & $0,10 \%$ & $0,10 \%$ & $0,30 \%$ & $0,60 \%$ & $0,40 \%$ \\
\hline
\end{tabular}

Fonte: IBGE

Assim, com os valores de $\alpha_{n}, m_{n, i}$ e $p_{i}$ resolvemos numericamente a equação acima que define o parâmetro de elasticidade de substituição.

\section{ERRATA:}

No artigo "Uma Nota Sobre a Oferta de EILD e Fechamento Vertical do Mercado de Banda Larga no Brasil", com número de DOI 10.5935/0034-7140.20150023, publicado no periódico Revista Brasileira de Economia, vol. 69(4):489-502, nas páginas 490 e 501:

Na página 490, onde se lia:

"Thompson \& Garbacz, 2018"

leia-se:

“Thompson \& Garbacz, 2008"

E na página 501, onde se lia:

“Thompson, H., \& Garbacz, C. (2018)"

leia-se:

“Thompson, H., \& Garbacz, C. (2008)” 